\title{
Olvidando el pasado: \\ la política familiar en España (1975-1996) ${ }^{1}$
}

Celia Valiente Fernández *

Desde 1975 la política familiar ${ }^{2}$ a nivel nacional en España se ha caracterizado por dos rasgos distintivos en comparación con otros países miembros de la Unión Europea (EU). Por una parte, ha implicado un volumen de gasto sumamente reducido, según se aprecia en la tabla 1. Por otra, no ha existido un Ministerio de la Familia o algún departamento ministerial de cierta importancia dedicado a asuntos familiares. Esta cuasi inexistencia de política familiar en España no puede ser explicada en relación con los índices de natalidad, los cuales han venido descendiendo sin interrupción en las últimas dos décadas, según muestra la tabla 2. Si bien en otros países las bajas tasas de natalidad han propiciado que las élites gobernantes establecieran políticas familiares con objetivos pronatalistas, ello no ha sucedido en España en las dos últimas décadas.

En este artículo argumento que la escasa relevancia de la política familiar desde 1975 es el resultado del rechazo a la establecida durante el régimen de Franco por parte de los principales actores políticos y sociales. Caracterizo la política familiar franquista como pronatalista y antifeminista, puesto que el crecimiento demográfico constituyó uno de sus principales objetivos, el cual pretendió alcanzarse, entre otros procedimientos, intentando apartar a las mujeres casadas del ámbito laboral y confinarlas al doméstico. La presencia de la política familiar en el discurso oficial y la propaganda del régimen fue tal que ha venido siendo recordada por los líderes sociales y políticos (y por la población en general) con posterioridad a 1975. Aquéllos han asociado desde entonces cualquier programa de promoción a la familia con los símbolos, las consignas y las medidas efectivas del período autoritario, por lo que han evitado tomar decisiones en este ámbito de actuación pública.

Es preciso destacar que este rechazo del legado del período autoritario no implicó el desmantelamiento de los programas familiares puestos en práctica durante el régimen de Franco, y que permanecían en vigor en 1975. Ello fue así debido al estilo político consensual característico de la transición a la democracia y de los años posteriores (SuBirats, 1992:129-130), el cual implicaba, de un lado, que la mayoría de las decisiones políti- cas se tomaron una vez que los distintos actores políticos y sociales hubieron alcanzado un acuerdo y, de otro, que, en general, intentaron evitarse las iniciativas políticas dirigidas exclusivamente contra la élite gobernante del anterior régimen 0 contra quienes lo apoyaron. Por consiguiente, las medidas franquistas dirigidas a las familias no se abolieron, toda vez que no se revisó la cuantía de las mismas. En consecuencia, su valor era ya meramente simbólico a mediados de los años ochenta, debido al efecto de la inflación.

En la primera parte de este trabajo presento la base teórica del argumento explicativo, e identifico las circunstancias en las que los decisores políticos tienden a rechazar la herencia del período autoritario. En la segunda parte contrasto las proposiciones teóricas en el caso empírico de la política familiar en España desde 1975.

\section{El argumento explicativo: el rechazo de la herencia del pasado}

Hugh Heclo (1974:315-18), al estudiar el proceso de toma de decisiones en materia de política social en Gran Bretaña y Suecia en el período contemporáneo, concluye que una política pública específica no es sólo la respuesta de las élites gobernantes a los problemas del momento, sino también el resultado de la modificación que éstos realizan a las políticas puestas en práctica con anterioridad (las cuales no fueron sino intentos gubernamentales de solucionar los mismos o similares problemas). Por su parte, Margaret WeIR y Theda SkOCPOL (1985:11921), en su examen sobre las respuestas de los gobiernos ante la depresión de los años treinta en Suecia, Gran Bretaña y Estados Unidos, parecen sugerir que cuando las élites políticas intentan resolver problemas tienden a introducir únicamente cambios 
menores en los programas existentes, en vez de tratar de instituir soluciones radicalmente distintas a las ejecutadas previamente. En otras palabras, según los tres autores mencionados, la existencia de medidas ya ensayadas en un área de actuación pública tiende a favorecer la continuidad en el proceso de toma de decisiones políticas.

Visto lo anterior, cabe preguntar si dicha continuidad también tendría lugar cuando se produjera un cambio de régimen político, por ejemplo, el paso de un sistema autoritario a uno democrático como el que tuvo lugar en España. Nancy Bermeo (1992:274-281) defiende que puede suceder lo contrario. Según BERMEO, las experiencias políticas vividas durante un período autoritario pueden contribuir a que los decisores políticos rechacen algunas prácticas y comportamientos fácilmente asociables con el autoritarismo. En esta línea, acuña el concepto de "aprendizaje político" (political learning) y lo define como "el proceso por el cual las personas modifican sus creencias y tácticas políticas como resultado de graves crisis, frustraciones y cambios dramáticos en el entorno" ${ }^{3}$. Añade que "el aprendizaje político consiste en el proceso de 'desacreditar' antiguas creencias".

Las proposiciones de Nancy Bermeo pueden ser desarrolladas intentando contestar al interrogante planteado por Peter Hall (1989:362) en los siguientes términos: "Si bien es acertado afirmar que las lecciones extraídas a partir de la experiencia política pasada ejercen cierta influencia sobre los decisores políticos, debe tenerse presente que las enseñanzas que la historia brinda son siempre ambiguas. Si ello es así, ipor qué son unas lecciones y no otras las aprendidas a partir de una experiencia política determinada?" En la línea trazada por Bermeo, e intentando responder al interrogante propuesto por Hall, argumento que, a diferencia de lo defendido por HEClo, WEIR y SKOCPOL (para los casos que ellos analizan, en los que no tiene lugar un cambio de régimen político) la existencia de medidas puestas en marcha con anterioridad puede favorecer discontinuidades en el proceso de toma de decisiones políticas, si se produce o se ha producido una transformación del régimen político. Planteo que las élites gobernantes post-autoritarias tienden a rechazar las actuaciones públicas del período anterior si confluyen las seis circunstancias siguientes:

(i) La política pública se originó en el período autoritario. Sobre todo en los casos en que las medidas se pusieron en práctica durante muchos años, los actores sociales y políticos del período democrático tienden a asociar cualquier programa sobre las mismas cuestiones con la política llevada a cabo en el pasado.

(ii) La política pública ocupó un lugar destacado en el discurso oficial, los rituales y las ceremonias del régimen autoritario. Siendo así, el recuerdo de dicha política permanece vivo en la memoria de los futuros actores políticos y sociales post- autoritarios, quienes posiblemente rechazarán las medidas pasadas en un intento de presentarse a sí mismos ante la población como líderes y/o representantes auténticamente democráticos. Cabe destacar que la posición preeminente de una política concreta en el discurso oficial y la propaganda del anterior régimen puede influir en el carácter de la futura política democrática en mayor medida que otros aspectos tradicionalmente considerados más importantes, tales como el volumen de recursos destinado a financiarla, o el número de personas a quienes afectó.

(iii) La política pública no fue puesta en práctica por una burocracia especialmente formada para ello, y que trabajaba en departamentos dedicados exclusivamente a su ejecución. Durante la transición a la democracia estos funcionarios "especialistas. podrían resistir la supresión de las instituciones donde tradicionalmente habían prestado sus servicios y oponerse a que los destinasen a otros departamentos (donde hubieran de adquirir habilidades con las que no contaban, además de ocuparse de nuevos cometidos).

(iv) La política pública del período autoritario puede ser percibida como contraria a los derechos de grupos de ciudadanos deseosos de incorporarse en pie de igualdad en el nuevo sistema político (tal sería el caso, por ejemplo, de las mujeres).

(v) No existen organizaciones de cierta importancia interesadas en el mantenimiento de la política pública.

(vi) El proceso de transición a la democracia se basa en la forja de un consenso entre los principales actores sociales y políticos. Es entonces probable que se evite tomar decisiones en aquellas áreas de actuación pública que pudieran ser fácilmente identificadas como las preferidas por las élites gubernamentales del régimen autoritario (durante el cual algunas de las futuras fuerzas políticas y sociales estuvieron ilegalizadas y fueron perseguidas).

\section{El caso empírico: la políica familiar en España (1975-1996)}

En esta sección cotejo las proposiciones formuladas en la anterior en el caso empírico de la política familiar en España en los últimos veinte años. En primer lugar presento una descripción de ésta antes y después de 1975. Más adelante, examino las posiciones y el comportamiento político de las organizaciones familiares, las asociaciones feministas, el feminismo institucional ${ }^{4}$, los principales partidos políticos y las confederaciones empresariales y sindicales durante las últimas dos décadas en relación con la promoción de la familia. 


\section{La política familiar en el régimen de Franco}

Según explico a continuación, las principales medidas de política familiar se diseñaron y pusieron en práctica en España durante el período franquista (no anteriormente) y ocuparon una posición destacada en la propaganda y el discurso oficial del régimen. Su gestión correspondió a una burocracia encargada de la política social en general o de otras cuestiones, esto es, no dedicada exclusivamente a la aplicación de la política familiar. Dado el carácter marcadamente antifeminista de esta última, pudo ser conceptualizada, entonces y más adelante, como contraria a las demandas de igualdad de las mujeres.

Permítaseme describir sucintamente los principales programas que compusieron la política familiar en la época de Franco. Las asignaciones monetarias por hijo a cargo, el llamado subsidio familiar, se instituyeron en 1938 y afectaron a la mayoría de los asalariados y los funcionarios. Su cuantía fue igual para todas las familias (excepto las numerosas, según explico más abajo) y aumentaba al hacerlo el número de hijos. No existieron niveles salariales máximos para recibir esta prestación, la cual fue financiada por el Estado, los empresarios y los trabajadores.

El plus de cargas familiares, establecido en 1945, consistió en un complemento salarial abonado a la mayor parte de los trabajadores, en una época en que las remuneraciones salariales eran bajas. Un porcentaje del total de los salarios pagados en una empresa (generalmente entre un 5 y un 25 por cien) se distribuía entre los trabajadores en función del número de hijos (si aquéllos mantenían económicamente a sus esposas, recibían una cantidad superior). Los empresarios financiaban casi por entero este programa, que por lo general estaba indiciado y era compatible con la percepción del subsidio familiar ${ }^{5}$.

En 1954 las prestaciones familiares existentes fueron sustituidas para casi todos los funcionarios por la llamada ayuda familiar, que consistía en asignaciones monetarias por hijos y esposa a cargo. ${ }^{6}$ El subsidio familiar, el plus de cargas familiares y la ayuda familiar eran pagados normalmente al "cabeza de familian, esto es, al esposo-padre asalariado, y únicamente en circunstancias excepcionales a la madre. Si ambos progenitores trabajaban, sólo uno (casi siempre el padre) recibía estas prestaciones.

Desde 1941 las parejas casadas podían beneficiarse de préstamos blandos cuya devolución se reducía con el nacimiento de cada hijo. La cuantía del préstamo a la que podían acceder se doblaba si la mujer trabajadora se comprometía a renunciar a su puesto de trabajo tras contraer matrimonio, y continuaba siendo ama de casa a menos que su marido quedara desempleado o sufriera una invalidez que le incapacitara para trabajar. En 1948 estos préstamos blandos fueron reemplazados por un pago monetario en concepto de matrimonio. También en 1941 se establecieron los premios nacionales y provinciales para las familias españolas con mayor número de hijos.
En lo que concierne a los programas diseñados para las familias numerosas, si bien algunos datan de 1926, fue durante el régimen de Franco cuando se instituyó la mayor parte de ellos. En 1943 las familias numerosas fueron definidas como aquéllas que mantenían a cuatro o más hijos. Las medidas dirigidas a ellas consistian principalmente en una cuantía más elevada del subsidio familiar, y en tratamiento preferencial en materia de impuestos, transporte público, préstamos, viviendas de promoción pública, tasas en centros educativos, empleo del cabeza de familia, adjudicación de explotaciones agrarias de tamaño familiar, y estancia en residencias, albergues, campamentos, balnearios y sanatorios públicos (Subsecretaría de Planificación de la Presidencia del Gobierno, 1977:318-20).

En 1966 el subsidio familiar y el plus de cargas familiares fueron sustituidos para la mayor parte de sus beneficiarios, por las prestaciones contributivas de la Seguridad Social. Como su nombre indica, las recibían los cotizantes al sistema de Seguridad Social, esto es, fundamentalmente los asalariados. Consistían en asignaciones mensuales por hijo y esposa a cargo de 200 y 300 pesetas respectivamente, así como asignaciones de pago único de 5.000 pesetas en concepto de matrimonio y de 2.500 pesetas por el nacimiento de cada hijo (Coll y MarTín, 1989:70). Como en el caso del subsidio familiar, su cuantía era la misma para todos sus perceptores, con independencia de su nivel de ingresos. De modo similar, las prestaciones eran abonadas al cabeza de familia y raramente a la madre. Si ambos esposos trabajaban, sólo uno (casi siempre el padre) tenía derecho a percibirlas, con la excepción del pago único en concepto de matrimonio que era recibido por ambos. Otra reforma de importancia en el ámbito de la política familiar tuvo lugar en 1968, cuando el programa de los funcionarios (la ayuda familiar) se convirtió en una asignación mensual por cada hijo a cargo menor de 10 años (200 pesetas), mayor de 10 años (300 pesetas) y por esposa a cargo (300 pesetas).

En cuanto a las exenciones fiscales por motivos familiares (hijos y/o esposa dependientes económicamente), se establecieron para la mayoría de los impuestos (por ejemplo, en el Impuesto sobre los Rendimientos del Trabajo Personal y el Impuesto General sobre la Renta de las Personas Físicas). Además, algunas desgravaciones fiscales especiales se instituyeron para las familias numerosas. Cabe resaltar que, por sorprendente que hoy parezca, ciertas exenciones aumentaban a medida que lo hacía el nivel de ingresos del contribuyente (Subsecretaría de Planificación de la Presidencia del Gobierno, 1977:323-325).

Hasta mediados de los años cincuenta o principios de los sesenta, la importancia económica de los programas familiares descritos en los párrafos precedentes fue notable, ya que constituyeron un considerable ingreso adicional para muchos asalariados. Es más, a la política familiar le correspondía la partida 
principal del gasto social del embriónico Estado de bienestar de la década de los cuarenta. No obstante lo anterior, desde los años sesenta y hasta 1975 el valor monetario de la mayor parte de las medidas raramente se elevó: por ejemplo, se incrementó sólo una vez (1971) el de las prestaciones contributivas de la Seguridad Social desde su creación en 1966, y sólo en dos ocasiones (1968 y 1975) el de la ayuda familiar desde su creación en 1954. Por consiguiente, debido al impacto de la inflación, el valor real de estos programas ya había comenzado a declinar antes de que comenzara la transición a la democracia (MeIL, 1995:63-72; Velarde, 1990:164-7).

La gestión de la política familiar no correspondió a una burocracia dedicada únicamente a estas cuestiones, sino que recayó en una multiplicidad de instituciones encargadas de la previsión social en general, o de otros cometidos. Muchos de los programas descritos se gestionaban en el Instituto Nacional de Previsión (INP) creado en 1908, antes de que existieran los programas familiares. El INP no era un organismo especializado en la promoción familiar, sino la principal institución en materia social (GuILlÉN, 1992:121). Debido al ya mencionado débil desarrollo del Estado de bienestar en los años cuarenta, la administración de la política familiar constituyó entonces una de las principales tareas del INP. Sin embargo, con el establecimiento de otras prestaciones sociales en los años siguientes, los programas familiares pasaron a ser tan sólo uno de los muchos ejecutados por el INP. De otro lado, el plus de cargas familiares se administraba independientemente en cada empresa por un comité de empresarios y trabajadores, mientras que la ayuda familiar se gestionaba en cada departamento por un comité de funcionarios. Por último, otras instituciones tenían a su cargo diversos programas de promoción familiar, entre otras, los Ministerios de Hacienda, Gobernación, Vivienda y Educación y Ciencia (Subsecretaría de Planificación de la Presidencia del Gobierno, 1977:315-316, 328).

El aumento de la población constituyó uno de los principales objetivos perseguidos por la política familiar franquista, meta que se pretendió alcanzar promocionando un tipo específico de familia: la originada por el matrimonio católico y formada por el mayor número posible de hijos (legítimos). La institución matrimonial fue entonces incentivada de diversas maneras, por ejemplo mediante los préstamos blandos de nupcialidad y los pagos únicos en concepto de matrimonio. Por otra parte, hasta los años setenta la mayoría de las prestaciones derivadas del mantenimiento de hijos a cargo se abonaba sólo cuando éstos eran legítimos. Finalmente, el alto número de hijos se recompensó con el incremento de la cuantía de las prestaciones familiares a medida que se elevaba el número de ellos, con los beneficios dirigidos a las familias numerosas, y los premios a nivel provincial y nacional para las más prolíficas.

La familia ideal según el discurso oficial del régimen consti- tuía una unidad jerárquica, donde la autoridad descansaba en el cabeza de familia, quien se suponía que sería el único aganador del pan. (o al menos el principal, si la familia atravesaba una adversa situación económica que hacía necesario que otros miembros realizaran trabajo extradoméstico). En este sentido, ciertas características de la política familiar contribuyeron a reforzar esta posición superior del esposo-padre, por ejemplo, la norma de que fuera él quien percibiera la mayor parte de las prestaciones, y que la madre sólo pudiera hacerlo en circunstancias excepcionales (NASH, 1991:172-3).

Este canon ideal también prescribía la existencia de una madre ama de casa. La maternidad fue definida como la principal obligación de las mujeres para con la familia, el Estado y la sociedad. Es más, fue considerada una función incompatible con otras, por ejemplo, el trabajo extradoméstico. Por esta razón, algunos programas familiares favorecieron explícitamente a las familias con un solo perceptor de ingresos. Tal fue el caso del plus de cargas familiares, cuya cuantía aumentaba considerablemente si el beneficiario mantenía económicamente a su esposa, o de la prestación contributiva de la Seguridad Social en concepto de esposa a cargo. Es preciso tener presente que, durante las dos primeras décadas del régimen, la política familiar fue puesta en marcha a la vez que se erigían numerosas barreras al trabajo extradoméstico de las mujeres, en especial de las casadas. Como ejemplos de estas últimas medidas pueden citarse las cláusulas de excedencia obligatoria de la trabajadora al contraer matrimonio, contenidas en un número elevado de ordenanzas laborales y reglamentaciones de trabajo que afectaban a empresas públicas y privadas, o la exigencia de que las casadas contaran con la autorización de su marido para firmar un contrato de trabajo o ejercer el comercio. Otras medidas, sin embargo, iban dirigidas a todas las mujeres y no sólo a las casadas; tal era el caso, entre otros, de las disposiciones que les prohibían desempenar determinados puestos en el sector público, en especial, los relacionados con la práctica jurídica ( $\mathrm{V}_{\mathrm{A}-}$ LIENTE FERNÁNDEZ, 1994:149-184).

La política familiar no fue en absoluto un conjunto de medidas encubiertas o poco publicitadas, sino que constituyó un tema recurrente en la retórica del régimen. Las preocupaciones demográficas fueron enfáticamente expuestas en discursos y escritos oficiales; de otro lado, la concesión de los premios de natalidad a las familias más prolíficas del país representó uno de los rituales más conocidos de la época, ya que los medios de comunicación de masas cubrieron ampliamente este evento, ofreciendo al público fotografías de las familias ganadoras, amplia información sobre las mismas y entrevistas a sus miembros. Por último, uno de los principales propósitos de las enseñanzas impartidas en el Servicio Social de la Mujer residía en persuadir a las jóvenes de que su principal misión residía en ser prolíficas madres y abnegadas esposas (Gallego, 1983:161-74) ${ }^{7}$. 


\section{La política familiar en España después de 1975}

Como explico en los párrafos siguientes, los decisores políticos han evitado, por lo general, la toma de decisiones respecto a la política familiar después de 1975 . El valor monetario de las medidas heredadas del franquismo no fue aumentado, por lo que su valor real era ya ínfimo a mediados de los ochenta por efecto de la inflación. En 1985 algunas de estas prestaciones fueron suprimidas (sobre todo, aunque no exclusivamente, las de marcado carácter antifeminista) mientras que las restantes continuaron languideciendo. Finalmente, en los años noventa, las que aún permanecían en vigor fueron convertidas en instrumentos contra la pobreza al ser destinadas exclusivamente a las familias con bajos o nulos ingresos.

Por lo que se refiere a las prestaciones contributivas de la Seguridad Social, su cuantía no fue elevada entre 1971 y 1985, momento en el que su importancia real era ya prácticamente simbólica $^{8}$. En 1985 fue suprimida la prestación de carácter antifeminista más visible: la asignación mensual por esposa a cargo. El argumento esgrimido por los decisores políticos fue que el Estado no debía promocionar el status de ama de casa ni desincentivar de ninguna otra manera el trabajo extradoméstico femenino. Las asignaciones de pago único por matrimonio y por el nacimiento de cada hijo fueron también abolidas. Por tanto, a partir de entonces la principal prestación familiar contributiva fue la asignación mensual por hijo a cargo de 250 pesetas. Es importante destacar que ya en 1986 la cuantía de esta asignación monetaria en concepto de hijo dependiente era más baja en España que en la mayoría de los países miembros de la UE. Es más, en estos últimos, y a diferencia de España, existían (y continúan existiendo) prestaciones familiares de otros tipos, por ejemplo, ciertos beneficios para familias monoparentales o los asociados al nacimiento de los hijos (Coll y Martín, 1989:72; RodRíGuez CABRERO, 1986).

La segunda y última reforma de las prestaciones familiares se llevó a cabo en los años noventa, con el propósito de convertir las prestaciones familiares, utilizadas anteriormente para promocionar a la familia, en instrumentos de la lucha contra la pobreza (Mell, 1994:975-6). Así, en 1990 se estableció el requisito de bajos ingresos familiares para percibir la prestación contributiva por hijo a cargo del sistema de la.Seguridad Social, cuya cuantía ascendió a 36.000 pesetas anuales. Además, se instituyó (también en el sistema de Seguridad Social) una prestación no contributiva por hijo a cargo (de 36.000 pesetas anuales) ${ }^{9}$. Por último, en 1992 los programas familiares de los funcionarios fueron suprimidos para la mayoría de ellos, pudiendo éstos recibir las citadas prestaciones familiares por hijo a cargo establecidas en 1990.

Al contrario de lo sucedido con el resto de la política familiar, los beneficios para las familias numerosas han sobrevivido durante las últimas dos décadas sin apenas modificaciones relevantes ${ }^{10}$. En la actualidad, su importancia económica es muy reducida, debido a la pérdida de su valor real como consecuencia de la inflación (puesto que su valor monetario se ha revisado al alza raramente), y al hecho de que cada vez existen menos familias de este tipo.

En cuanto a las exenciones fiscales, el regresivo sistema impositivo franquista fue reemplazado por un nuevo impuesto sobre la renta, el Impuesto sobre la Renta de las Personas Físicas (IRPF), de carácter progresivo. Hasta el momento, los contribuyentes del IRPF han podido beneficiarse de tres exenciones fiscales relacionadas con su situación familiar: por matrimonio (hasta 1987), por cada hijo dependiente, y por gastos de custodia de hijos menores de tres años en determinadas circunstancias (desde 1992)" 11

Al igual que sucedió con las prestaciones contributivas, las exenciones fiscales de características antifeministas más destacadas fueron suprimidas. Desde 1978 era obligatoria la tributación conjunta (ambos esposos debían pagar juntos el IRPF). En 1989 el Tribunal Constitucional sentenció que este tipo de tributación (tal como estaba establecido en España) era inconstitucional, puesto que desincentivaba en muchos casos el trabajo extradoméstico femenino. Esta tributación penalizaba a los núcleos familiares formados por más de un perceptor de ingresos, ya que el del segundo perceptor (generalmente la mujer) frecuentemente incrementaba el nivel de ingresos familiares hasta situarlo en un tramo impositivo superior. Desde 1989 las parejas casadas pueden elegir entre la tributación conjunta y la individual (según esta última, cada individuo de la unidad familiar tributa por sus ingresos con independencia de los de los otros miembros).

En el ámbito de la política familiar, la importancia económica de las exenciones fiscales es en la actualidad mayor que la de las transferencias monetarias. Gerardo MeIL (1994:976-979) ha calculado la de estas desgravaciones, de las prestaciones a que se refiere la tabla 1, y de otras medidas no consideradas en este artículo, como el subsidio recibido por las mujeres trabajadoras durante el período de descanso por maternidad y los gastos médicos asociados con la maternidad. Mell concluye que las exenciones fiscales no compensan el bajo nivel de gasto social en materia familiar en España reflejado en la tabla 1, si bien reducen algo la distancia con respecto a otros países miembros de la UE.

En síntesis, los programas familiares más importantes en España a medidados de los años noventa son de tres tipos: en primer lugar, las prestaciones de la Seguridad Social por hijo a cargo en sus modalidades contributiva y no contributiva para familias de bajos ingresos, administradas por el organismo encargado de la gestión de las prestaciones económicas de la Seguridad Social (el Instituto Nacional de la Seguridad Social, de- 
pendiente del Ministerio de Trabajo y Seguridad Social); en segundo lugar, las desgravaciones para los contribuyentes del IRPF gestionadas por el Ministerio de Economía y Hacienda; y, por último, los beneficios para las familias numerosas, administrados en distintos departamentos ministeriales. En términos comparativos, la política familiar se caracteriza por un bajo nivel de gasto y por una marcada invisibilidad institucional, puesto que ningún Ministerio o departamento ministerial de cierta entidad se denomina "de la Familia" o de modo similar ${ }^{12}$.

\section{Los actores sociales y políticos en el área de la política familiar después de 1975}

En general, los principales actores sociales y políticos han evitado toda iniciativa en el área de la política familiar después de 1975. Explico a continuación que esta inactividad es debida a sus intentos de distanciarse del período autoritario, para lo cual examino sus posiciones acerca de la promoción de la familia, así como sus comportamientos en este ámbito de actuación pública en algunos momentos cruciales.

\section{(i) Las organizaciones familiares}

En algunos países miembros de la UE las organizaciones familiares son capaces de influir en el proceso de toma de decisiones políticas debido, entre otras razones, a su elevado número de miembros y a su acceso a importantes foros políticos. Así por ejemplo, en los Países Bajos, Bélgica y Francia estas asociaciones participan en órganos consultivos en el área de la política familiar (Dumon, 1990:363-4). En la España de después de Franco las organizaciones familiares han sido el principal actor que ha apoyado una política familiar más amplia, si bien su influencia ha sido mínima, debido a que su número de miembros es muy bajo y a que la mayoría de estos grupos está organizada a nivel local y provincial, careciendo de plataformas a nivel nacional efectivas para ejercer presión en la esfera política (IGLESIAS, 1994:540-2).

\section{(ii) El movimiento feminista}

Por lo que se refiere al movimiento de mujeres ${ }^{13}$, muchos grupos de este tipo se formaron en España a finales de los años sesenta y principios de los setenta, y junto con otras organizaciones (la mayoría de ellas ilegales) participaron en la oposición al régimen autoritario (SCANLON, 1990:94). Las militantes feministas rechazaron entonces los principios que inspiraron a los decisores políticos franquistas, así como las políticas sustantivas en el área de la promoción de la familia y en otras.

Las feministas españolas han realizado pocas aportaciones a la teoría feminista internacional, y han adquirido la mayor parte de sus premisas teóricas leyendo traducciones de algunas obras escritas por autoras de otros países (Threlfall, 1985:59-60). En general, y con excepciones importantes, las teóricas feministas internacionales han dedicado notables esfuerzos a refutar las concepciones tradicionales de la familia que consideran esta institución como la que proporciona a los individuos (con independiencia de su edad y sexo) las mayores gratificaciones y posibilidades de desarrollo personal. Estas autoras han destacado que la familia es uno de los ámbitos donde las mujeres gozan de una posición inferior, puesto que frecuentemente dependen económicamente de los hombres, realizan la mayor parte de trabajos domésticos y de cuidados y, en ocasiones, se convierten en víctimas por excelencia de la violencia doméstica (EISENSTEIN, 1991:86-96). Las feministas españolas recibieron estas conceptualizaciones, y con ayuda de ellas analizaron la experiencia histórica franquista y/o confirmaron sus análisis previos sobre la misma. De este modo concluyeron que la mejor política familiar (según se define en este artículo) era una nopolítica, argumentando que el Estado no debía promover una institución donde la posición de las mujeres era en muchos casos de subordinación. En cualquier caso, la influencia del movimiento feminista sobre el diseño de políticas públicas del Estado central es escasa en términos comparativos, debido, entre otros motivos, a su reducido número de militantes, su limitada capacidad de iniciar debates públicos y de atraer la atención de los medios de comunicación de manera continua, y su alto grado de fragmentación (no compensado por la existencia de plataformas importantes a nivel nacional) ${ }^{14}$.

\section{(iii) El feminismo institucional}

España cuenta desde finales de los años setenta con ainstituciones feministasn, si bien en este artículo examino sólo el Instituto de la Mujer (IM), fundado en 1983, por ser el organismo de igualdad del Estado central más importante ${ }^{15}$.

La mayoría de las feministas de Estado, al igual que muchas militantes del movimiento feminista, han coincidido en su rechazo a la política familiar franquista y, por extensión, a cualquier política familiar (el IM no cuenta con un departamento o sección encargada de asuntos familiares). Esta coincidencia no es sorprendente, si se tiene en cuenta que algunas de las primeras feministas institucionales habían formado parte anteriormente del movimiento de mujeres. De otro lado, algunas de estas primeras feministas de Estado, junto con otras personas que participaron en el establecimiento del IM, eran miembros del Partido Socialista Obrero Español (PSOE) o se encontraban muy cercanas a este partido político. Como describo a continuación, el PSOE ha prestado poca o ninguna atención al asunto de la promoción de la familia mediante el establecimiento de políticas públicas. 


\section{(iv) Los partidos políticos}

Respecto al partido en el Gobierno entre 1977 y 1982, Unión de Centro Democrático (UCD), cabría esperar que sus programas y actuaciones reflejaran un vivo interés por los asuntos familiares, tal como ha sucedido en el caso de numerosos partidos de centro y conservadores en otros países miembros de la UE. Sin embargo, a pesar de su color político UCD no propugnó una política familiar más intensa, según se desprende de la poca atención que los asuntos familiares recibieron en sus programas electorales y, más adelante, en su actuación política.

Los programas electorales de UCD contenían tan sólo una o dos afirmaciones acerca de la familia como institución básica del conjunto social. (UCD, 1977:13), o como el acentro de formación de los hijos y escuela de solidaridad y convivencian (UCD, 1979:25). De otro lado, hacían escasas o nulas referencias a la promoción de la familia mediante políticas públicas.

Fue precisamente durante el período de gobierno de UCD cuando la cuantía de las prestaciones familiares disminuyó radicalmente en términos reales, debido a que su valor monetario no se elevó en este período caracterizado por las altas tasas de inflación. Por otra parte, entre 1977 y 1982 se perdieron ciertas oportunidades de organizar un amplio debate sobre la política familiar, ya que ni UCD ni ningún otro actor social o político relevante parecía interesado en iniciar o participar en el mismo.

Una de estas oportunidades apareció en las discusiones sobre la reforma del sistema de la Seguridad Social mantenidas desde mediados de los años setenta por representantes de los partidos políticos, las confederaciones empresariales y sindicales, y algunos altos funcionarios y académicos (GUILLÉn, 1992:1278). Pese a que las prestaciones familiares contributivas formaban parte de la Seguridad Social, los documentos gubernamentales que contribuyeron al desarrollo del mencionado debate sólo contenían referencias de pasada a dichas prestaciones. Normalmente afirmaban que su cuantía era sumamente reducida, y que las marcadamente antifeministas (por ejemplo, las asignaciones mensuales por esposa a cargo) habían de ser suprimidas. En ningún momento se hizo un análisis de la política familiar, discutiendo la deseabilidad de sus objetivos, la adecuación de sus medios, y los resultados obtenidos ${ }^{16}$.

En cuanto al PSOE, la política familiar no ha constituido en absoluto una prioridad de esta formación política durante las dos últimas décadas, según muestran sus programas electorales, que apenas contienen referencia alguna a la promoción de la familia (PSOE, 1977, 1979, 1982, 1986, 1989, 1993, 1996).

Las militantes feministas del PSOE han sido particularmente activas en sus intentos de evitar la toma de decisiones en este área de actuación pública ${ }^{17}$. Argumentaban que las mujeres habian sido tradicionalmente asociadas con el cuidado de los niños y de cualquier otro familiar que necesitara atenciones, lo que había contribuido a su confinamiento en el ámbito doméstico. Las militantes socialistas querían actuar políticamente en beneficio de las mujeres, entendidas éstas como individuos independientes, y no como miembros de la unidad familiar ${ }^{18}$. La estrategia que recomendaron entonces al partido consistía en el establecimiento de políticas de igualdad de oportunidades para las mujeres, a fin de reducir las diferencias entre éstas y los hombres en ámbitos tan diversos como el mercado laboral, el sistema educativo o la arena política. Implícitamente apoyaron la renuncia a una política familiar intensiva que, a su juicio, reforzaría directa o indirectamente las obligaciones familiares de mujeres. Estas recomendaciones fueron importantes y son mencionadas aqui no porque las propusiese un sector minoritario del partido, sino porque terminaron convirtiéndose en la política sustantiva del PSOE en el área de la familia y de la mujer.

Una de las responsables de la Secretaría de la Mujer del PSOE, Matilde Fernández Sanz, fue nombrada Ministra de Asuntos Sociales en 1988, cuando se creó el Ministerio. Uno de los cometidos de éste residía en el establecimiento de políticas públicas dirigidas a ciudadanos en circunstancias particularmente desfavorables, como las mujeres, los inmigrantes o los minusválidos. A la vista de lo explicado con anterioridad no sorprende que ninguna sección de este Ministerio se encargara específicamente de asuntos familiares. La citada Ministra afirmó claramente en varias ocasiones que la política social debía dirigirse a los individuos, y sólo excepcionalmente a las familias (FERnÁndez SANZ, 1989:96), declaraciones corroboradas por la segunda Ministra de Asuntos Sociales (1993-1996), Cristina Alberdi, conocida abogada feminista antes de iniciar su carrera política (El País 20 diciembre 1995:28).

Existe suficiente evidencia empírica para afirmar que el PSOE apenas ha prestado atención a la política familiar. Por ejemplo, los documentos gubernamentales elaborados a partir de 1982 como contribución a la ya mencionada discusión sobre la reforma de la Seguridad Social sólo hacen alguna mención de pasada a los programas familiares, normalmente repitiendo que debían suprimirse los marcadamente antifeministas, y sugiriendo que la política familiar debería dirigirse únicamente a los núcleos familiares de bajos ingresos ${ }^{19}$. De hecho, la supresión de los programas antifeministas realizada en 1985 junto con la conversión de las asignaciones mensuales por hijo a cargo en instrumentos contra la pobreza a principios de los años noventa constituyen las dos únicas excepciones de importancia en la pauta general de inactividad por parte del PSOE en el área de la política familiar.

En lo que concierne al Partido Popular (PP, previamente bajo los nombres de Alianza Popular, Coalición Democrática y Coalición Popular - AP, CD , CP), el principal partido de la oposición desde 1982 hasta 1996, su modesto papel en la toma de decisiones políticas en materia de políica familiar resulta particularmente intrigante. Sus programas electorales contienen un ma- 
yor número de referencias al tema que nos ocupa que los del PSOE (AP, 1977;16, 34; 1982, 135-136; CD, 1979:7, 11-12, 21-22, 37-39; CP, 1986:55; PP, 1989:28-30, 60; 1993:96-98; 1996:181183). Además, en algunos documentos escritos y conferencias su anterior Presidente, Manuel Fraga Iribarne (1977:97-101; 1984), ha afirmado que la familia es una institución básica de la sociedad, a la vez que abogaba por el establecimiento de más activas medidas para promover aquélla.

Pese a lo anterior, cuando se examinan las actuaciones de los representantes del PP, se comprueba que han prestado bastante menos atención a la política familiar de lo que sugiere la lectura de sus programas electorales. Por ejemplo, en cuanto a la actividad parlamentaria del PP en el Congreso de los Diputados entre 1977 y principios de 1996, estos diputados han presentado muy pocas proposiciones de ley relacionadas con la elevación de la cuantía de las prestaciones familiares, y sólo en raras ocasiones han planteado preguntas parlamentarias al Gobierno. Cabe destacar que gran parte de esta actividad parlamentaria se produjo en la década de los noventa, al ser propiciada por un acontecimiento internacional: la declaración por parte de las Naciones Unidas de 1994 como Año Internacional de la Familia.

La reacción ante el pasado autoritario constituye la clave para entender por qué las élites políticas del PP han actuado de modo diferente en el tema de la familia a como lo han hecho muchos partidos del mismo color político en otros países miembros de la UE. Desde la transición a la democracia el PP ha intentado presentarse ante el electorado como un nuevo partido político auténticamente democrático, y no como el heredero del franquismo, empresa que no ha resultado sencilla, entre otros factores porque algunos líderes del PP, entre ellos su anterior Presidente, Manuel Fraga Iribarne, habían sido destacadas figuras políticas durante el régimen anterior. Las élites del PP han evitado entonces cuidadosa y sistemáticamente la utilización de símbolos y la actividad pública en materias que pudieran fácilmente ser asociadas con el pasado autoritario, tales como la promoción de la familia.

Para concluir este análisis del discurso público y del comportamiento de los líderes de los principales partidos políticos, es necesario recordar que en España no se ha constituido ningún partido democristiano de importancia, debido sobre todo a que la Iglesia no apoyó la formación de tal organización política. En otros países han sido precisamente los partidos democristianos los principales (si bien no los únicos) promotores de una amplia e intensa política familiar.

\section{(v) Las confederaciones empresariales y sindicales}

Al igual que el resto de los actores políticos y sociales ya examinados, y por las mismas razones, la Confederación Española de
Organizaciones Empresariales (CEOE) y las confederaciones sindicales Unión General de Trabajadores (UGT) y Comisiones Obreras (CCOO) han evitado cualquier actividad en el área de la política familiar. En primer lugar, apenas discutieron estos asuntos cuando participaron en los debates sobre la reforma de la Seguridad Social antes citados, según puede comprobarse en los documentos con los que contribuyeron a los mismos (CEOE, 1981; 1982:13; 1983; 1985; Círculo de Empresarios, 1979:14, 7274; JimÉnEZ, 1982) ${ }^{20}$. En segundo lugar, los pactos sociales firmados por los representantes de los empresarios y/o de los trabajadores y/o del Estado, tales como el Acuerdo Marco Interconfederal (1980), el Acuerdo Nacional de Empleo (1981), el Acuerdo Interconfederal (1983) o el Acuerdo Económico y Social (1984), además de los llamados "pactos de la Moncloa" firmados por los partidos políticos, no contenían ninguna referencia a la política familiar ${ }^{21}$. Por último, en general, el establecimiento de prestaciones familiares no ha sido contemplado en la negociación colectiva (Álvarez y MárQuez, 1994:201; CASAS, 1977; CASTINEEIRA, 1989:1023; Del Rey y Durán, 1993:884; Gallardo, 1991:483).

\section{Conclusión}

En este artículo he explicado por qué, en comparación con otros países miembros de la UE, España dedica muy pocos recursos a la política familiar a nivel nacional. Esta especificidad española puede entenderse teniendo en cuanta la influencia de la memoria histórica. Desde 1975, los principales actores políticos y sociales han intentado distanciarse lo más posible de la política familiar puesta en marcha durante la época de Franco, de marcado carácter pronatalista y antifeminista, y ampliamente presente en el discurso y la propaganda oficial del régimen. Estos actores han asociado cualquier medida en el ámbito de la promoción de la familia con los programas implantados durante el régimen autoritario, lo que les ha hecho evitar participar en la toma de decisiones en torno a estos asuntos. Debido al estilo consensual de la actividad política característico de la transición y consolidación de la democracia en España, las medidas heredadas de la época franquista no fueron desmanteladas. En ausencia de una burocracia organizada en su defensa, languidecieron durante una década o más, debido al impacto de la inflación.

Similitudes entre el Estado de bienestar en España y en otros países occidentales han sido ya enfatizadas por la literatura de ciencias sociales (ESPING-ANDERSEN, 1995; GUILLÉN, 1990, 1992). Sin negar que, en general, ambos Estados de bienestar comparten más elementos comunes que diferenciadores, uno de estos últimos reside ciertamente en el escaso peso de la política familiar en España, lo cual también puede afirmarse para los restantes países del Sur de Europa. 
Muchas investigaciones sobre políticas sociales (no sólo relativas a la promoción de la familia) en la España democrática son aún necesarias; por el momento, cabe sospechar que un rechazo de las medidas sociales puestas en marcha durante el período franquista se ha producido en circunstancias en que confluyeron las seis condiciones identificadas en este artículo: cuando la política se originó en el pasado autoritario, ocupó un destacado lugar en la propaganda del régimen, no fue administrada por una burocracia especializada y dedicada exclusivamente su gestión, su permanencia amenazó los derechos de grupos de ciudadanos

\begin{tabular}{|c|c|c|}
\hline \multicolumn{3}{|c|}{$\begin{array}{l}\text { Tabla } 1 \\
\text { Gasto social en familia, como proporción del total del } \\
\text { gasto social en paises miembros de la Unión Europea } \\
(1980,1991)\end{array}$} \\
\hline \multicolumn{3}{|c|}{\begin{tabular}{l|l|l} 
Pais & 1980 & 1991
\end{tabular}} \\
\hline Bélgica & 10,7 & 8,0 \\
\hline Dinamarca & 9,9 & 10,3 \\
\hline Francia & 10,7 & 8,2 \\
\hline Alemania & 9,0 & 6,0 \\
\hline Grecia & 3,6 & 1,4 \\
\hline Irlanda & 8,0 & 10,6 \\
\hline Italia & 6,9 & 3,6 \\
\hline Luxemburgo & 8,8 & 9,5 \\
\hline Países Bajos & 8,9 & 5,5 \\
\hline Pontugal & 6,8 & 5,4 \\
\hline España & 2,7 & 0,6 \\
\hline Reino Unido & 11,4 & 9,6 \\
\hline Europa 12 & 9,2 & 6,4 \\
\hline
\end{tabular}

Fuente. Comisión de las Comunidades Europeas (1993). Social Protection Expenditure and Receipts, 1980-1991. Luxemburgo: Office for Official Publication of the European Communities.

Nota: El gasto social bajo la categoría familia. no corresponde exactamente con la politica familiar examinada en este artículo. Por una parte, sólo las transferencias monetarias están incluidas en la tabla, mientras que este trabajo también examina las desgravaciones fiscales. Por otra, este trabajo estudia sólo los programas dirigidos a familias compuestas por uno o dos progenitores y sus hijos dependientes, mientras que la tabla incluye otros relativos a otros familiares, por ejemplo, personas de la tercera edad. Ello no obstante, la tabla sirve (por ejemplo, las mujeres) que aspiraran a ser incluidos en pie de igualdad en el nuevo sistema político, ningún actor social de importancia defendió su permanencia, y hubo de sobrevivir en un período democrático dominado por un estilo político consensual. Esta hipótesis puede ser contrastada con la evidencia empírica para España en el caso de otras políticas sociales distintas de la familiar, o con respecto a las medidas de promoción a la familia en Italia, Portugal y Grecia, gobernadas con anterioridad por regímenes políticos autoritarios, y en las que la política familiar tiene también muy poca importancia (tabla 1).

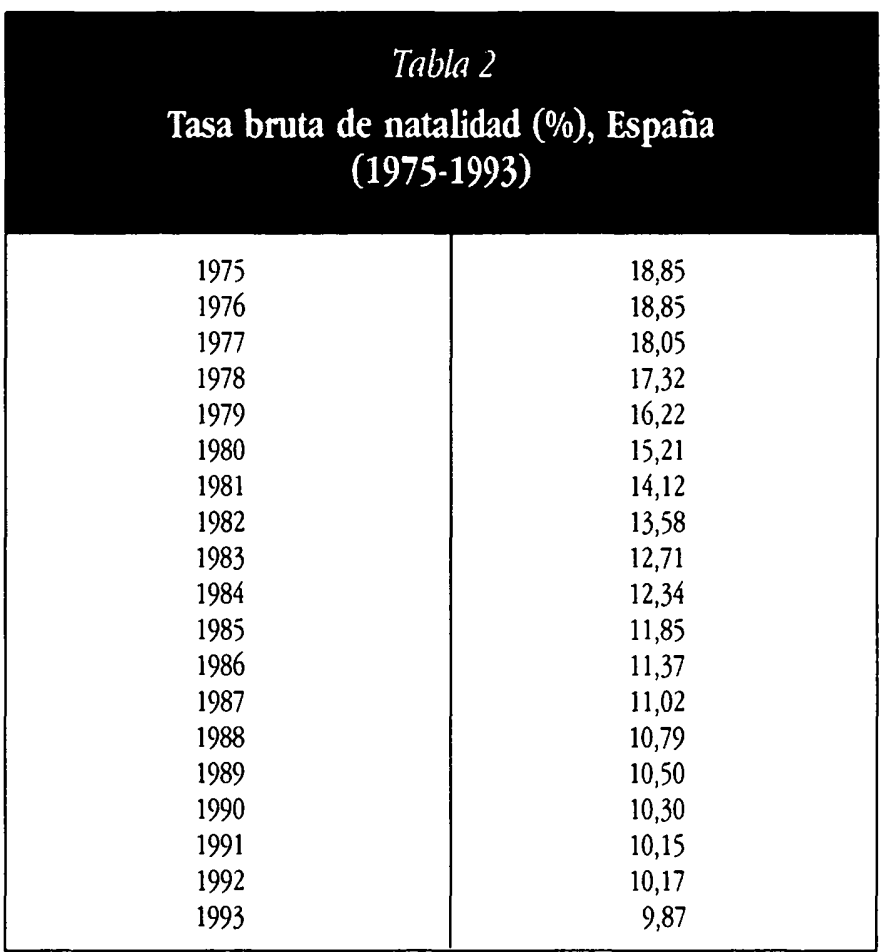

Fuente. Instituto Nacional de Estadística. Movimiento natural de la población española. Madrid: Instituto Nacional de Estadistica.

para mostrar que el gasto social en materia familiar en relación con el gasto social total ha sido más bajo en España que en el resto de los paises miembros de la UE desde los años setenta. Por último, es importante destacar que, dado que en algunos paises la politica de promoción familiar se compone de multitud de programas de poca imporancia económica gestionados por muchas instituciones distintas, cabe sospechar que algunos no se tuvieran en cuenta al construir la tabla. 
- Universidad Autónoma de Madrid. Facultad de Ciencias Económicas y Empresariales. Departamento de Sociología y Antropología Social. Ciudad Universitaria de Canto Blanco (Madrid).

' Desearía agradecer a Miguel Beltrín, Juan J. Linz, Isabel Madruga, Ana Rico y Juan Carlos Rodriguez sus inestimables comentarios a borradores de este articulo, los cuales fueron presentados en: el Primer Congreso Español de Ciencia Politica y de la Administración (Bilbao, 5-7 diciembre 1994), el congreso Comparative Research on Welfare State Reforms organizado por la International Sociological Association (Researcb Committee 19: Poverty, Social Welfare and Social Policy) (Pavia, 14-17 septiembre 1995), la Euroconference on Social Policy in an Environment of Insecurity de la European Association for the Advancement of Social Sciences y el Interdisciplinary Center for Comparative Research in the Social Sciences (Lisboa, 811 noviembre 1995) y el seminario Comparing Social Welfare Systems in Southern Europe organizado por la Mission Recberche Expérimentation (MIRE) (Florencia, 22-24 febrero 1996). Una versión algo diferente apareció en inglés en la revista South European Society \& Politics (1996, Vol.1, No.1:95-114) publicada por Frank Cass (Londres)

2 Utilizo la definición de política familiar acuñada por Jane JENSON y Mariette SINEAU (1994:24): gastos directos e indirectos realizados por el Estado (en forma de transferencias monetarias o desgravaciones fiscales) para atender a las familias y sus necesidades. Esta definición no incluye otra dimensión de esta política, esto es, los derechos y obligaciones de los familiares establecidos por las disposiciones legislativas relativas al matrimonio, el divorcio y la herencia, que habrán de ser objeto de un trabajo posterior. Por limitaciones de espacio y recursos de investigación, y dada la escasez de fuentes secundarias, en este artículo examino la política familiar elaborada por el Estado central y dirigida a los núcleos familiares formados por uno o dos padres y sus hijos. Analizo las medidas que considero más importantes, a saber, las que han afectado a un número significativo de personas $y / o$ han constituido innovaciones respecto a programas anteriores.

${ }^{3}$ La traducción al castellano de textos publicados en otro idioma ha sido realizada para este artículo por Celia Valiente Fernández.

4 Instituciones cuyo principal cometido es impulsar la formulación y puesta en práctica de medidas para alcanzar un mayor grado de igualdad entre las mujeres y los hombres, se han fundado y consolidado en la mayor parte de los países occidentales desde aproximadamente los años setenta. En numerosas ocasiones, además, se han establecido organismos similares de considerable importancia en los ámbitos regional y/o local. En la literatura de ciencias sociales este conjunto de

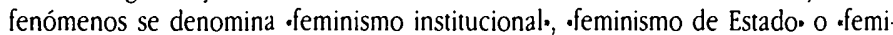
nismo oficial. (STETSON y MAZUR, 1995). El -organismo de igualdad• más importante en España a nivel nacional es el Instituto de la Mujer (Val.ente. Fernández, 1996).

s El plus de cargas familiares se conocía popularmente como los •puntos:, dado que los familiares que dependían económicamente de los trabajadores reporraban a éstos puntos, en función de los cuales percibían una parte del porcentaje de los salarios destinado a financiar este programa.

${ }^{6}$ Los funcionarios facultativos, técnicos, administrativos o auxiliares recibían las siguientes sumas mensuales por cada hijo a cargo menor de 10 años, mayor de 10 años y esposa a cargo: 200, 300 y 300 pesetas respectivamente. Los funcionarios subalternos recibian 160,240 y 240 pesetas por los mismos conceptos

7 El Servicio Social de la Mujer, administrado por la Sección Femenina (primero dependiente de Falange y más tarde del Movimiento) comprendía desde 1940 dos partes: tres meses de enseñanza política y doméstica, junto con otros tres meses de trabajo obligatorio y gratuito en dependencias estatales, generalmente de carácter asistencial. Debían realizarlo las españolas entre 17 y 35 años si querían obtener títulos de estudios, trabajar en empresas y organismos públicos, en empresas concesionarias del Estado o que recibían subvenciones de éste, ser profesionales colegiados o matricularse en la universidad (entre otras circunstancias).

8 En 1971, las asignaciones mensuales por hijo y esposa a cargo ascendian al 6,13 por cien y 9,19 por cien del salario mínimo interprofesional ( 4.080 pesetas al mes) respectivamente. En 1985 estos porcentajes eran 0,62 y 0,93 por cien (del salario mínimo de 40.140 pesetas) (Coll y MarTín, 1989:70).

9 En diciembre de 1994 eran 759.999 las familias que recibían prestaciones familiares por hijo a cargo en la modalidad contributiva, mientras que 175.850 lo hacian en la no contributiva (Instituto Nacional de la Seguridad Social, 1996:386).

10 En noviembre de 1995 se reconoció a los núcleos familiares de tres hijos la condición de familias numerosas. A mediados de 1996, ello significaba que podian beneficiarse de la reducción de las tasas universitarias, pero aún no del resio de las medidas destinadas a este lipo de familias.

1 El número de contribuyentes que se benefició de la exención fiscal por matrimonio aumentó anualmente, pasando de 4.853 .989 en 1981 a 5.835 .659 en 1987, último año en que existió esta desgravación fiscal. El número de los afectados por reducciones fiscales en concepto de hijos a cargo también se incrementó (pasando de 3.679 .989 en 1981 a 5.710 .668 en 1993), como también lo hizo el número de contribuyentes. Por último, en 1993, 106.299 contribuyentes se acogieron a exenciones fiscales por custodia de hijos menores de 3 años. Estos datos proceden de Ministerio de Economía y Hacienda (1981-1995)

1" Un análisis de la política familiar en los países miembros de la UE puede consultarse en HaNtrals y LeTABLIER (1996:136-189).

13 Este epígrafe sobre el movimiento feminista está basado en SCAnlon (1990), Threlfall (1985) y 7 entrevistas en profundidad realizadas a militantes para VALIFNTE FERNÁNDEZ (1996)

${ }_{14}$ En 1994, el Instituto de la Mujer había registrado 106 organizaciones de mujeres (no necesariamente feministas) de ámbito nacional, y 2346 de ámbito regional o local (Vallente FernándeZ, 1996:193).

is Los dos principales documentos programáticos del IM $(1987,1993)$ son los dos planes de igualdad de oportunidades para las mujeres, que constituyen la principal fuente para la redacción de este epígrafe, junto con entrevistas en profundidad realizadas a feministas de Estado para Valiente. Ffrnáindez (1996).

16 Véanse, por ejemplo: Presidencia del Gobierno $(1982: 11,17)$ y .Propuesta de medidas de racionalización y mejora de la Seguridad Social. (1982:11, 17). Un resumen del debate mencionado en el texto sobre la reforma de la Seguridad Social puede consultarse en VELARDE (1990:291-354).

${ }^{17}$ Las feministas socialistas han venido ganando estatus organizativo dentro del partido. Así, en 1976 formaron el colectivo •Mujer y Socialismo•, que en un principio era poco más que un círculo de estudio y de debate dependiente de la Secretaría de Formación. En 1981 uno de sus miembros, Carmen Mestre, fue elegida vocal de la Comisión Ejecutiva Federal del partido, y otras la siguieron en años posteriores. Finalmente, en diciembre de 1984 lograron que :Mujer y Socialismo. se convirtiera en una Secretaría Ejecutiva de la Comisión Ejecutiva Federal (Threlfall, 1985:4849).

${ }_{18}$ Entrevista personal (13 abril 1994) con Matilde Fernández Sanz, Ministra de Asuntos Sociales entre 1988 y 1993.

19 Véanse, por ejemplo, Gonzalo, Ferreras y Tejerina (1985), Ministerio de Trabajo y Seguridad Social (1985:40-1, 137-8) y Secretaria General de la Seguridad Social (1991).

${ }^{20}$ Estas aportaciones de las confederaciones sindicales al mencionado debate pueden consultarse en Papeles de Economia Española número especial seguridad Social. (Documentos). de 1982. La poca importancia conferida por los agentes sociales a la política familiar se refleja en la total ausencia de referencias al tema en sus principales publicaciones periódicas. Al consultar todos los números de CEOE: BOletin Informativo (diciembre 1980-noviembre 1995) y Unión de UGT (junio 1981noviembre 1995) no he encontrado ninguin artículo sobre el asunto en consideración aquí.

${ }^{21}$ Los pactos citados pueden consultarse en DE LA VILLA (1985). 


\section{Bibliografia}

Áliarez, J. Y MARQuez, A. (1994): 'La Seguridad Social y los convenios colectivos', En F. NAVARRO (ed.), XI Jomadas universitarias andaluzas de Derecbo del Trabajo y relaciones laborales, Madrid: Trotta y Consejo Andaluz de Relaciones laborales, pp.179-206.

AP (1977): Qué es Alianza Popular, Madrid: Alianza Popular.

AP (1982): Elecciones generales: programa electoral.

Bermeo, N. (1992): 'Democracy and the Lessons of Dictatorship', Comparative Politics, abril, pp.273-291.

CASAS, M. (1977): Autonomia colectiva y Seguridad Social, Madrid: Instituto de Estudios Fiscales.

CASTiNizira, J. (1989): 'La protección a la familia en la negociación colectiva 19871988', Relaciones Laborales, II, pp.1023-1034.

CD (1979): Elecciones generales: programa electoral.

CEOE (1981): 'Consideraciones sobre la financiación de la Seguridad Social en España', Informes y Estudios de CEOE, 7, junio-julio, pp.13-17.

CEOE (1982): 'Análisis del programa de mejora y racionalización de la Seguridad Social', Informes y Estudios de CEOE, 16, mayo, pp.9-18.

CEOE (1983): 'La reforma de la Seguridad Social desde el punto de vista empresarial', Informes y Estudios de CEOE, 26, octubre-noviembre, pp.15-36.

CEOE (1985): 'Reflexiones sobre la reforma de la Seguridad Social', Informes $y$ Estudios de CEOE, 35, marzo-abril, pp.21-24.

CEOE: Boletin Informativo diciembre 1980-noviembre 1995.

Círculo de Empresarios (1979): Documento Base para el estudio de un modelo de Seguridad Social en España, Madrid: Círculo de Empresarios.

Coll, P. y MarTí, R. (1989): 'La protección de la maternidad, de la familia y de la vivienda', Revista de Economia y Sociologia del Trabajo, 3, pp.67-83

CP (1986): Elecciones generales: programa electoral.

DE LA VILLA, L. (ed.) (1985): Los grandes pactos, acuerdos y convenios colectivos a partir de la transición democrática: antologia, Madrid: Ministerio de Trabajo y Seguridad Social.

Del ReY, S. y Durán, G. (1993): 'Las mejoras voluntarias: análisis de la reciente negociación colectiva y jurisprudencia, Civitas: Revista Española de Derecho del Trabajo, 62, noviembre-diciembre, pp.867-914.

Dumon, W. (1990): 'Family Policy in the EEC Countries: A General Overview', En W. Dumon (ed.), Family Policy in EEC Countries, Luxemburgo: Office for Official Publications of the European Communities, pp.351-369.

Eisenstein, H. (1991): Gender Shock: Practicing Feminism on Two Continents, Sydney: Allen \& Unwin.

El País 20 diciembre 1995:28.

ESPING-ANDERSEN, G. (1995): 'Welfare States Without Work: The Impasse of Labor Shedding and Familialism in Continental European Social Policy', Centro de Estudios Avanzados en Ciencias Sociales Estudios/Working Papers, 71.

Ferníndez SAnz, M. (1989): 'Protección social y política de igualdad: Estado y sociedad', Cuadernos de Acción Social, 16, pp.94-101.

FraGa Iribarne, M. (1977): Alianza Popular, Bilbao: Albia.

Fraga Irubarne, M. (1984): 'La familia y la política', En Fundación Cánovas del Castillo (ed.), Jornada Nacional sobre la Familia, Madrid: Fundación Cánovas del Castillo, pp.61-67.

Gallardo, R. (1991): 'Las mejoras complementarias del sistema de la Seguridad Social en la negociación colectiva', Relaciones laborales 7 (23), pp.8-23.

Gallego, M. (1983): Mujer, Falange y Franquismo, Madrid: Taurus.

Gonzalo, B.; FerReras, F. y Tejerina, J. (1985): Evolución y tendencias de la Seguridad Social durante la crisis económica, Madrid: Ministerio de Trabajo y Seguridad Social.

GUlluÉn, A. (1990): 'The Emergence of the Spanish Welfare State (1876-1923): the Role of Ideas in the Policy Process', Centro de Estudios Avanzados en Ciencias Sociales Estudios/Working Papers, 10.

Gulltén, A. (1992): 'Social Policy in Spain: From Dictatorship to Democracy (1939-
1982)', En Z. FERGE y J. KolBERG (eds.), Social Policy in a Cbanging Europe, Frankfun am Main y Boulder (Colorado): Campus Verlag y Westview Press, pp.119-142.

Hau, P. (1989): 'Conclusion: the Politics of Keynesian Ideas', En P. Hau (ed.), Tbe Political Power of Economic Ideas: Keynesianism Acrass Nations, Princeton (New Jersey): Princeton University Press, pp.361-391.

HaNTRAIS, L. y Letabler, M.T. (1996): Families and Family Policies in Europe, Londres y Nueva York: Longman.

Heclo, H. (1974): Modern Politics in Britain and Sweden: From Relief to Income Maintenance, New Haven (Connecticut): Yale University Press.

IGLESIAS, J. (1994): 'La políica familiar durante la transición', En Fundación Foessa (ed.), VInforme sociológico sobre la situación social en España, Madrid: Fundación Foessa, pp. $525-542$.

Instituto de la Mujer (1987): I Plan para la lgualdad de Oportunidades de las Mujeres 1988-1990, Madrid: Instituto de la Mujer.

Instituto de la Mujer (1993): II Plan para la lgualdad de Oportunidades de las Mujeres 1993-1995, Madrid: Instituto de la Mujer.

Instituto Nacional de la Seguridad Social (1996): Memoria 1994, Madrid: Instituto Nacional de la Seguridad Social.

Jenson, J. y Sinfau, M. (1994): 'Family Policy and Women's Citizenship in Mitterrand's France' Trabajo presentado en el congreso Crossing Borders: International Dialogues on Gender, Social Politics and Citizenship, Estocolmo, mayo 27-29.

JimÉNEZ, J. (1982): 'Situación y reforma de la Seguridad Social', En Fundación Friedrich Ebert (ed.), La reforma de la Seguridad Social, Madrid: Fundación Friedrich Ebert, pp.193-204.

MEIL, G. (1994): 'L'évolution de la politique familiale en Espagne', Population, 4-5, pp.959-984.

MEIL, G. (1995): 'La política familiar española durante el franquismo', Revista Internacional de Sociologia, 11, mayo-agosto, pp.47-87.

Ministerio de Economía y Hacienda (1981-1995): Memoria de la Administración Tributaria, Madrid: Ministerio de Economía y Hacienda.

Ministerio de Trabajo y Seguridad Social (1985): Documento base sobre la reforma de la Seguridad Social, Madrid: Ministerio de Trabajo y Seguridad Social.

NASH, M. (1991): 'Pronatalism and Motherhood in Franco's Spain', En G. Bock y P. Thane (eds.), Maternity and Gender Policies: Women and the Rise of the European Welfare States, 1880s-1950s, Londres: Routledge, pp.160-177.

Papeles de Economia Española, número extraordinario 'Seguridad Social (Documentos)' (1982).

PP (1989; 1993; 1996): Elecciones generales: programas electorales.

Presidencia del Gobierno (1982): La Seguridad Social española: programa de mejora y racionalización, Madrid: Presidencia del Gobierno.

'Propuesta de medidas de racionalización y mejora de la Seguridad Social', Papeles de Economia Española, número extraordinario 'Seguridad Social (Documentos)' (1982), pp.1-19.

PSOE (1977; 1979; 1982; 1986; 1989; 1993; 1996): Elecciones generales: programas electorales.

Rodrícuez Cabrero, G. (1986): 'Bienestar social y familia en España', Cuadernos de Acción Social, 2 (noviembre), pp.33-49.

SCANlon, G. (1990): 'El movimiento feminista en España, 1900-1985: logros y dificultades', En J. Astelarra (ed.), Participación Politica de las Mujeres, Madrid: Centro de Investigaciones Sociológicas y Siglo XXl, pp.83-100.

Secretaría General de la Seguridad Social (1991): Estudios preparatorios para la Ley de prestaciones no contributivas, Madrid: Ministerio de Trabajo y Seguridad Social. SteTson, D. y Mazur, A. (eds.) (1995): Comparative State Feminism, Thousind Oaks (California): Sage.

SUBIRatS, J. (1992): Un problema de estilo: la formación de políticas piiblicas en España, Madrid: Centro de Estudios Constitucionales.

Subsecretaría de Planificación de la Presidencia del Gobierno, (1977): Acción protectora y esinuctura orgánica de la Seguridad Social, Madrid: Subsecretaría de Planificación de la Presidencia del Gobierno. 
Threlfall, M. (1985): 'The Women's Movement in Spain', New Left Review, 151, pp. $44-73$

UCD (1977; 1979): Elecciones generales: programas electorales.

Unión junio 1981-noviembre 1995.

Valuente Fernández, C. (1994): Politicas puiblicas para la mujer trabajadora en Italia y España (1900-1991), Madrid: Centro de Estudios Avanzados en Ciencias Sociales/Instituto Juan March de Estudios e Investigaciones.

VALIENTE FernándeZ, C. (1996): 'El feminismo institucional en Espaǹa: el Instituto de la Mujer, 1983-1994', Revista Internacional de Sociologia, 13, enero-abril, pp.163204.

VELARDE, J. (1990): El tercer viraje de la Seguridad Social en España, Madrid: Instituto de Estudios Económicos.

WeIR, M. y Sxocpot, T. (1985): 'State Siructures and the Possibilities for -Keynesian. Responses to the Great Depression in Sweden, Britain, and the United States', En P. Evans, D. RuesChEMeYER y T. SKOCPOL (eds.), Bringing the State Back In, Cambridge: Cambridge University Press, pp.107-163. 